Discrete ANALYsis, 2017:17, 10 pp.

www.discreteanalysisjournal.com

\title{
Set Reconstruction on the Hypercube
}

\author{
Luke Pebody \\ Received 3 September 2016; Published 27 October 2017
}

\begin{abstract}
Given an action of a group $G$ on a set $S$, the $k$-deck of a subset $T$ of $S$ is the multiset of all subsets of $T$ of size at most $k$, each given up to translation by $G$.

For a given subset $T$, the reconstruction number of $T$ is the minimum $k$ such that the $k$-deck uniquely identifies $T$ up to translation by $G$, and the reconstruction number of the action $G: S$ is the maximum reconstruction number of any subset of $S$.

The concept of reconstruction number extends naturally to multisubsets $T$ of $S$ and in [2], the author calculated the multiset-reconstruction number of all finite abelian groups. In particular, it was shown that the multiset-reconstruction number of $\mathbb{Z}_{2}^{n}$ was $n+1$. This provides an upper bound of $n+1$ to the reconstruction number of $\mathbb{Z}_{2}^{n}$. The author also showed a lower bound of $\left\lfloor\frac{n+1}{2}\right\rfloor$ in the same paper.

The purpose of this note is to close the gap. The reconstruction number of $\mathbb{Z}_{2}^{n}$ is
\end{abstract}

$$
\left\lfloor n+1-\log _{2}\left(n+1-\log _{2}(n)\right)\right\rfloor .
$$

Key words and phrases: reconstruction

\section{Introduction and Definitions}

Given a set $S$ and non-negative integer $k$, denote by $S^{(k)}$ the set of subsets of $S$ of size $k$. Given an action on a group $G$ of a set $S$, the $k$-deck of a subset $T$ is the multiset of all subsets of $T$ of size $k$, each given up to translation by $G$ :

$$
\operatorname{deck}_{k}(T)=\left\{\{g U: g \in G\}: U \in T^{(k)}\right\} .
$$

Say that subsets $T_{1}$ and $T_{2}$ are $k$-indistinguishable if $\operatorname{deck}_{i}\left(T_{1}\right)=\operatorname{deck}_{i}\left(T_{2}\right)$ for all $i \leq k$ and $k$ distinguishable otherwise. In particular, for any subset $T$ of $G$, any element $g$ of $G$ and any integer $k, g T$ and $T$ are $k$-indistinguishable and sets $T_{1}$ and $T_{2}$ are $k$-indistinguishable if and only for all $i \leq k$, there is a bijection $\phi_{i}: T_{1}^{(i)} \rightarrow T_{2}^{(i)}$ such that for all $U \in T_{1}^{(i)}$ there exists a $g$ such that $\phi_{i} U=g U$. 


\section{LUKE PEBODY}

For subsets $T_{1}$ and $T_{2}$ say that the distinguishing number of $T_{1}$ and $T_{2}$, denoted by $d_{G: S}\left(T_{1}, T_{2}\right)$, is the smallest number $k$ such that $T_{1}$ and $T_{2}$ are $k$-distinguishable. If there is no such $k$ (and so $T_{1}$ and $T_{2}$ are translates), the distinguishing number is $\infty$.

For a subset $T$ of $S$, say that the reconstruction number $r_{G: S}(T)$ is the smallest number $k$ such that for any subset $U$ of $S$, if $T$ and $U$ are $k$-indistinguishable then $U$ is a translate $g T$ of $T$, and the reconstruction number $r(G: S)$ is the maximum value of $r_{G: S}(T)$ for any subset $T \subseteq S$.

One can extend the definition of reconstruction from sets to multisets. Let a multiset from $S$ be a map $\phi: S \rightarrow \mathbb{N}$ from $S$ to the non-negative integers $\mathbb{N}$, and then define the $k$-deck of $\phi$ to be the map $\operatorname{deck}_{k} \phi: S^{k} \rightarrow \mathbb{N}$ defined by

$$
\operatorname{deck}_{k} \phi\left(s_{1}, s_{2}, \ldots, s_{k}\right)=\sum_{g \in G} \phi\left(g s_{1}\right) \phi\left(g s_{2}\right) \ldots \phi\left(g s_{k}\right) .
$$

Given this definition of a $k$-deck, we can define $k$-distinguishable and $k$-indistinguishable as before, and for a multiset $\phi$ from $G$, say that the multiset reconstruction number $\operatorname{rm}_{G: S}(\phi)$ is the smallest number $k$ such that for any multiset $\psi$ from $G$, if $\phi$ and $\psi$ are $k$-indistinguishable, then $\psi$ is a translate $g \phi$ (defined by $g \phi(s)=\phi\left(g^{-1} s\right)$ ) of $\phi$. Finally, define the multiset reconstruction number $\operatorname{rm}(G: S)$ to be the maximum value of $\operatorname{rm}_{G: S}(\phi)$ for any multiset $\phi$ from $G$.

One of the first general results in this area was in [1], where the authors showed that the reconstruction number of a group action $r(G: S)$ was bounded above by $\log _{2}(|G|)$.

In this paper we will focus on the action of an abelian group acting on itself by multiplication. For the specific case of the cyclic group acting on itself by multiplication, it was proved in [3] that the reconstruction number of $r\left(Z_{n}\right)$ was at most equal to 9 times the number of prime factors of $n$ and was equal to 3 for prime $n$. This was improved upon in [2] where the multiset reconstruction number was calculated for every abelian group and, thereby, it was shown that $r\left(\mathbb{Z}_{n}\right) \leq 6$.

We will focus on the group $\mathbb{Z}_{2}^{n}$ which we will identify both, where necessary, with the $n$-dimensional vector space over the field of 2 elements and with the power set of the $n$ element set. In [2], it was shown that the multiset reconstruction number of $\mathbb{Z}_{2}^{n}$ is $n+1$, from which it follows that $r\left(\mathbb{Z}_{2}^{n}\right) \leq n+1$. In the same paper, a lower bound was provided of $\frac{n+1}{2}$. In this paper we will show that the reconstruction number is equal to $\left\lfloor n+1-\log _{2}\left(n+1-\log _{2}(n)\right)\right\rfloor$. This expression may seem a tad unwieldy, but comes from the following fact.

Theorem 1. For positive integers $n$ and $k$, the statements

$$
k \leq\left\lfloor n+1-\log _{2}\left(n+1-\log _{2}(n)\right)\right\rfloor
$$

and

$$
2^{n+1-k} \geq k
$$

are equivalent.

Proof. Let us suppose that $t$ is the unique non-negative integer that $2^{t}+t \leq n<2^{t+1}+t+1$. Then if we set $k=n-t$, we see that $2^{n+1-k}=2^{t+1} \geq n-t=k$, but if we set $k=n+1-t$, we see that $2^{n+1-k}=2^{t}<n+1-t=k+1$. Since $2^{n+1-k}$ is decreasing in $k$, it follows that $2^{n+1-k} \geq k$ if and only if $k \leq n-t$. 


\section{SET RECONSTRUCTION ON THE HyPERCUBE}

Further, if $2^{t}+t \leq n<2^{t+1}$, then $\log _{2}(n)$ is between $t$ and $t+1$, so $n+1-\log _{2}(n)$ is between $2^{t}$ and $2^{t+1}$. Similarly, if $2^{t+1} \leq n<2^{t+1}+t+1$, then $\log _{2}(n)$ is between $t+1$ and $t+2$, so $n+1-\log _{2}(n)$ is between $2^{t}$ and $2^{t+1}$.

It follows that $\left\lfloor n+1-\log _{2}\left(n+1-\log _{2}(n)\right)\right\rfloor=n-t$.

In Section 2, we will show that if $2^{n+1-k} \geq k$ then the reconstruction number of $\mathbb{Z}_{2}^{n}$ is at least $k$, and in Sections 3 and 4, we will show the converse.

\section{Lower Bound}

To prove our lower bound, we provide a method for creating sets which are not easily distinguishable.

Theorem 2. Suppose that $G$ contains a subgroup $H$, and that set $A$ is the union of sets $A_{1}, \ldots, A_{k}$ such that for each $i, A_{i}$ is contained in a distinct coset $g_{i} H$ of $H$.

Suppose likewise that set $B$ is the union of sets $B_{1}, \ldots, B_{k}$ such that for each $i, B_{i}$ is contained in a distinct coset $g_{i}^{\prime} H$ of $H$.

Suppose finally that for each $1 \leq i \leq k, A \backslash A_{i}$ and $B \backslash B_{i}$ are translates. Then $A$ and $B$ are not $k$-1-distinguishable.

Proof. Let $T$ be a subset of $G$ of size smaller than $k$, and for set $S \subseteq G$ denote by $f(S)$ the number of elements $g$ of $G$ for which $g T \subseteq S$.

Note that $f(S)$ counts the number of occurrences of the orbit $\{g T: g \in G\}$ of $T$ in $\operatorname{deck}_{|T|}(S)$. To show that $A$ and $B$ are not $k-1$-distinguishable, it is therefore sufficient to show that for any such $T$, $f(A)=f(B)$.

Let $t$ be the number of distinct cosets of $H$ in which the elements of $T$ lie. Note that $t \leq|T|<k$. Then for any $g$, the elements of $g T$ lie in $t$ cosets of $H$. Therefore each translate $g T$ contained in $A$ is contained in exactly $k-t$ of the sets $A \backslash A_{j}$, so $(k-t) f(A)=\sum_{i} f\left(A \backslash A_{i}\right)=\sum_{i} f\left(B \backslash B_{i}\right)=(k-t) f(B)$ and hence $f(A)=f(B)$.

Since this is true for all sets $T$ of size less than $k$, it follows that $A$ and $B$ are not $(k-1)$-distinguishable.

Now we show how to find sets satisfying the properties of Theorem 2

Corollary 3. For positive integer $k \geq 3$, suppose that abelian group $G$ contains a subgroup $H$ of index at least $k$.

Suppose also that subgroup $H$ has $k$ subgroups $H_{1}, H_{2}, \ldots, H_{k}$ with cosets $h_{1} H_{1}, h_{2} H_{2}, \ldots, h_{k} H_{k}$ such that the cosets have an empty intersection, but the intersection of any $k-1$ of them is not empty.

Then the reconstruction number of $G$ is at least $k$.

Proof. Since $\bigcap_{i} h_{i} H_{i}$ is different from $\bigcap_{i \neq j} h_{i} H_{i}$ for all $j$, it follows that the cosets $h_{i} H_{i}$ are distinct. Further, since for all pairs $i, j, h_{i} H_{i} \cap h_{j} H_{j} \neq \emptyset$ and cosets of a single subgroup are equal or disjoint, it follows that the subgroups $H_{i}$ and $H_{j}$ are distinct.

Choose $k$ distinct cosets $g_{1} H, g_{2} H, \ldots, g_{k} H$ of $H$ in $G$, and then for $1 \leq i \leq k$, let $A_{i}=g_{i} H_{i}$ and let $B_{i}=g_{i} h_{i} H_{i}$. Finally let $A$ be the disjoint union of the $A_{i}$ and $B$ be the disjoint union of the $B_{i}$. 
Choose $1 \leq i \leq k$, and let $x$ be any element of $\bigcap_{j \neq i} h_{j} H_{j}$. Then for all $j \neq i, x A_{j}=x g_{j} H_{j}=g_{j} x H_{j}=$ $g_{j} h_{j} H_{j}=B_{j}$, and so $x\left(A \backslash A_{i}\right)=\bigcup_{j \neq i} x A_{j}=\bigcup_{j \neq i} B_{j}=B \backslash B_{i}$.

Thus the sets $A_{i}$ and $B_{i}$ satisfy the condition of Theorem 2, and hence $A$ and $B$ are not $k-1$ distinguishable. Further, we will show that $A$ and $B$ are not translates.

Suppose otherwise, then there exists $g \in G$ such that $g A=B$. Since cosets of $H$ are preserved by the map $x \rightarrow g x$, it follows that this map must map each $A_{i}$ to some $B_{j}$. However, if $i \neq j$ then $A_{i}$ and $B_{j}$ are cosets of different subgroups $H_{i}$ and $H_{j}$ which means one cannot be mapped to the other.

Thus for each $i$, it follows that $g g_{i} H_{i}=g_{i} h_{i} H_{i}$, from which it follows (since $G$ is abelian) that $g \in h_{i} H_{i}$ for all $i$. This is a contradiction, as

$$
\bigcap_{i} h_{i} H_{i}=\emptyset
$$

It follows that $A$ and $B$ are not $k-1$-distinguishable, and are not translates, so $G$ must have reconstruction number of at least $k$.

To conclude we will show the existence of cosets with this intersection property.

Lemma 4. The hypercube $\mathbb{Z}_{2}^{k-1}$ contains $k$ cosets of subgroups with empty intersection, such that the intersection of any $k-1$ of them is not empty.

Proof. Representing the elements of $\mathbb{Z}_{2}^{k-1}$ as sequences $\left(x_{1}, x_{2}, \ldots, x_{k-1}\right)$ of elements of $\mathbb{Z}_{2}$, we can take our $k$ cosets to be $\left\{x: x_{1}=0\right\},\left\{x: x_{1}=x_{2}\right\},\left\{x: x_{2}=x_{3}\right\}, \ldots,\left\{x: x_{k-2}=x_{k-1}\right\}$ and $\left\{x: x_{k-1}=1\right\}$.

Clearly no vector satisfies all of these conditions, but if you remove any one condition, the rest can be satisfied.

This concludes the work for the lower bound.

Corollary 5. If positive integers $n, k$ have $2^{n+1-k} \geq k$ then the reconstruction number of $\mathbb{Z}_{2}^{n}$ is at least $k$.

Proof. For $k \geq 3$, this follows directly from Corollary 3 and Lemma $4 . \mathbb{Z}_{2}^{k-1}$ is a subset of $\mathbb{Z}_{2}^{n}$ of index $2^{n+1-k}$, so if this is at least $k$, the conditions of Lemma 4 apply.

For $k=1$, the condition is $2^{n} \geq 1$, which is equivalent to $n \geq 0$. Clearly, for any $n$, the 0 -deck does not distinguish between \{\} and $\mathbb{Z}_{2}^{n}$ which are distinct, so $\mathbb{Z}_{2}^{n}$ has reconstruction number at least 1 .

For $k=2$, the conditions is that $2^{n-1} \geq 2$, which is equivalent to $n \geq 2$.

For $n \geq 2$ and for any two non-zero elements $a, b$ of $\mathbb{Z}_{2}^{n}$ the sets $\{0, a\}$ and $\{0, b\}$ are non-translates that are not 1-distinguishable so $\mathbb{Z}_{2}^{n}$ has reconstruction number at least 2 .

\section{The Fourier Transform}

Given elements $x=\left(x_{1}, x_{2}, \ldots, x_{n}\right)$ and $y=\left(y_{1}, y_{2}, \ldots, y_{n}\right)$ of $\mathbb{Z}_{2}^{n}$, define $\langle x, y\rangle=x_{1} y_{1}+x_{2} y_{2}+\ldots+$ $x_{n} y_{n}$. Then, given a mapping $f: \mathbb{Z}_{2}^{n} \rightarrow \mathbb{Z}$ the Fourier Transform $\hat{f}: \mathbb{Z}_{2}^{n} \rightarrow \mathbb{Z}$ is defined by $\hat{f}(x)=$ $\sum_{y \in \mathbb{Z}_{2}^{n}} f(y)(-1)^{\langle x, y\rangle}$.

Given a multiset $f$ from $\mathbb{Z}_{2}^{n}$ and a linear map $\theta: \mathbb{Z}_{2}^{n} \rightarrow \mathbb{Z}_{2}^{k}$, denote by $\theta f$ the multiset from $\mathbb{Z}_{2}^{k}$ defined by

$$
\theta f(x)=\sum_{z: \theta z=x} f(z)
$$


For every linear map $\theta: \mathbb{Z}_{2}^{n} \rightarrow \mathbb{Z}_{2}^{k}$, there is a dual map $\theta^{*}: \mathbb{Z}_{2}^{k} \rightarrow \mathbb{Z}_{2}^{n}$ with the property that for every $x \in \mathbb{Z}_{2}^{n}$ and $y \in \mathbb{Z}_{2}^{k},\langle\theta x, y\rangle=\left\langle x, \theta^{*} y\right\rangle$. The dual map gives a clean description of the Fourier Transform of linear images of multisets.

Lemma 6. Given a multiset $f$ from $\mathbb{Z}_{2}^{n}$ and a linear map $\theta: \mathbb{Z}_{2}^{n} \rightarrow \mathbb{Z}_{2}^{k}$ with dual $\theta^{*}$, the Fourier Transform of the image $\theta f$ is given by

$$
\widehat{\theta f}(x)=\hat{f}\left(\theta^{*} x\right)
$$

Proof.

$$
\begin{aligned}
\widehat{\theta f}(x) & =\sum_{y \in \mathbb{Z}_{2}^{k}} \theta f(y)(-1)^{\langle x, y\rangle} \\
& =\sum_{y \in \mathbb{Z}_{2}^{k}} \sum_{z: \theta z=y} f(z)(-1)^{\langle x, y\rangle} \\
& =\sum_{z \in \mathbb{Z}_{2}^{n}} f(z)(-1)^{\langle x, \theta z\rangle} \\
& =\sum_{z \in \mathbb{Z}_{2}^{n}} f(z)(-1)^{\left\langle\theta^{*} x, z\right\rangle} \\
& =\hat{f}\left(\theta^{*} x\right)
\end{aligned}
$$

The effect of translation on the Fourier Transform is similarly easy to describe.

Lemma 7. Given a multiset $f$ from $\mathbb{Z}_{2}^{n}$ and an element $z \in \mathbb{Z}_{2}^{n}$, the Fourier Transform of the translate $z+f$ is given by

$$
\widehat{(z+f)}(x)=\hat{f}(x)(-1)^{\langle x, z\rangle} .
$$

Proof.

$$
\begin{aligned}
\widehat{(z+f)(x)} & =\sum_{y \in \mathbb{Z}_{2}^{n}}(z+f)(y)(-1)^{\langle x, y\rangle} \\
& =\sum_{y \in \mathbb{Z}_{2}^{n}} f(z+y)(-1)^{\langle x, y\rangle} \\
& =\sum_{y^{\prime} \in \mathbb{Z}_{2}^{n}} f\left(y^{\prime}\right)(-1)^{\left\langle x, z+y^{\prime}\right\rangle} \\
& =\sum_{y^{\prime} \in \mathbb{Z}_{2}^{n}} f\left(y^{\prime}\right)(-1)^{\langle x, z\rangle}(-1)^{\left\langle x, y^{\prime}\right\rangle} \\
& =(-1)^{\langle x, z\rangle} \sum_{y^{\prime} \in \mathbb{Z}_{2}^{n}} f\left(y^{\prime}\right)(-1)^{\left\langle x, y^{\prime}\right\rangle} \\
& =(-1)^{\langle x, z\rangle} \hat{f}(x) .
\end{aligned}
$$


It is proved in [2] that the reconstruction number of a given multiset can be described in terms of the Fourier Transform.

Theorem 8. The $k$-deck of $f$ gives exactly the same information as knowing all values $\prod_{i=1}^{k} \hat{f}\left(x_{i}\right)$ for all sequences $\left(x_{1}, x_{2}, \ldots, x_{k}\right)$ with $x_{1}+x_{2}+\ldots+x_{k}=0$.

We can use this to show the effect of linear maps on distinguishability.

Theorem 9. For any multisets $f, g$ on $\mathbb{Z}_{2}^{n}$ and integer $k \geq 2$ :

1. If $f$ and $g$ are $k$-indistinguishable then for any linear map $\theta$ from $\mathbb{Z}_{2}^{n}$ to any target space, $\theta f$ and $\theta g$ are $k$-indistinguishable.

2. If $f$ and $g$ are $k$-distinguishable, then there exists a linear map $\theta: \mathbb{Z}_{2}^{n} \rightarrow \mathbb{Z}_{2}^{k-1}$ such that $\theta f$ and $\theta g$ are $k$-distinguishable.

Proof. By Theorem $8, f$ and $g$ being $k$-indistinguishable is equivalent to

$$
\hat{f}\left(x_{1}\right) \ldots \hat{f}\left(x_{i}\right)=\hat{g}\left(x_{1}\right) \ldots \hat{g}\left(x_{i}\right)
$$

for all sequences $x_{1}, \ldots, x_{i}$ with $i \leq k$ and $x_{1}+\ldots+x_{i}=0$.

Suppose $f$ and $g$ are $k$-indistinguishable, and let $\theta: \mathbb{Z}_{2}^{n} \rightarrow \mathbb{Z}_{2}^{p}$ be any linear map. Then by Lemma 6 , for any sequence $x_{1}, \ldots, x_{i} \in \mathbb{Z}_{2}^{p}$ with $i \leq k$ and $x_{1}+\ldots+x_{i}=0$,

$$
\begin{aligned}
\widehat{\theta f}\left(x_{1}\right) \ldots \widehat{\theta f}\left(x_{i}\right) & =\hat{f}\left(\theta^{*} x_{1}\right) \ldots \hat{f}\left(\theta^{*} x_{i}\right) \\
& =\hat{g}\left(\theta^{*} x_{1}\right) \ldots \hat{g}\left(\theta^{*} x_{i}\right) \\
& =\widehat{\theta g}\left(x_{1}\right) \ldots \widehat{\theta g}\left(x_{i}\right),
\end{aligned}
$$

so $\theta f$ and $\theta g$ are $k$-indistinguishable.

Similarly, for $k$-distinguishable $f$ and $g$, there exists a sequence $x_{1}, \ldots, x_{i}$ with $i \leq k, x_{1}+\ldots+x_{i}=0$ and

$$
\hat{f}\left(x_{1}\right) \ldots \hat{f}\left(x_{i}\right) \neq \hat{g}\left(x_{1}\right) \ldots \hat{g}\left(x_{i}\right) .
$$

Then define map $\theta: \mathbb{Z}_{2}^{n} \rightarrow \mathbb{Z}_{2}^{k-1}$ in terms of its dual by $\theta^{*} e_{j}=x_{j}$ for $j<i$ and $\theta^{*} e_{j}=0$ for $j \geq i$. Note that $\theta^{*}\left(e_{1}+\ldots+e_{i-1}\right)=x_{i}$.

Thus if we let $y_{j}=e_{j}$ for $j<i$ and $y_{i}=e_{1}+\ldots+e_{i-1}$, then $y_{1}, \ldots, y_{i}$ are a sequence with $i \leq k$, $y_{1}+\ldots+y_{i}=0$ and

$$
\widehat{\theta f}\left(y_{1}\right) \ldots \widehat{\theta f}\left(y_{i}\right) \neq \widehat{\theta g}\left(y_{1}\right) \ldots \widehat{\theta g}\left(y_{i}\right),
$$

so $\theta f$ and $\theta g$ are $k$-distinguishable.

\section{Structure of maximally indistinguishable multisets on $\mathbb{Z}_{2}^{k-1}$}

For this section, we will investigate the nature of pairs of multisets from $\mathbb{Z}_{2}^{k-1}$ which have distinguishing number $k$. We will give concrete examples of such multisets now. 
For distinct non-negative integers $a, b$ and positive integers $a_{1}, \ldots, a_{k-1}$, denote by $f_{(a, b),\left(a_{1}, \ldots, a_{k-1}\right)}$ the multiset defined by

$$
f\left(\left(x_{1}, x_{2}, \ldots, x_{k-1}\right)\right)=\left\{\begin{array}{l}
a+\sum_{i} a_{i} x_{i} \text { if } \sum x_{i} \text { is even } \\
b+\sum_{i} a_{i} x_{i} \text { if } \sum x_{i} \text { is odd }
\end{array}\right.
$$

We note that the Fourier Transform of $f_{(a, b),\left(a_{1}, \ldots, a_{k-1}\right)}$ takes a particularly simple form. Since the all-ones vector $e_{1}+e_{2}+\ldots+e_{k-1}$ will come up quite a lot this section, denote it by $h$.

Theorem 10. The Fourier Transform of $f_{(a, b),\left(a_{1}, \ldots, a_{k-1}\right)}$ is given by

$$
\hat{f}_{(a, b),\left(a_{1}, \ldots, a_{k-1}\right)}(x)=\left\{\begin{array}{l}
2^{k-2}\left(a+b+\sum a_{i}\right) \text { if } x=0 \\
2^{k-2} a_{i} \text { if } x=e_{i} \\
2^{k-2}(a-b) \text { if } x=h \\
0 \text { otherwise }
\end{array}\right.
$$

Proof. We can decompose $f_{(a, b),\left(a_{1}, \ldots, a_{k-1}\right)}$ as the linear combination of $\frac{a+b}{2}$ parts of the function $x \rightarrow 1$, $\frac{a-b}{2}$ parts of the function $x \rightarrow(-1)^{\langle h, x\rangle}$ and $a_{i}$ parts each of the function $x \rightarrow x_{i}$.

Each part has a Fourier Transform that is trivial to compute.

The Fourier Transform of $x \rightarrow 1$ is

$$
z \rightarrow \sum_{y \in \mathbb{Z}_{2}^{k-1}}(-1)^{\langle z, y\rangle}
$$

which is $2^{k-1}$ for $z=0$ and 0 otherwise.

The Fourier Transform of $x \rightarrow(-1)^{\langle h, x\rangle}$ is

$$
z \rightarrow \sum_{y \in \mathbb{Z}_{2}^{k-1}}(-1)^{\langle h, y\rangle\langle z, y\rangle}=\sum_{y \in \mathbb{Z}_{2}^{k-1}}(-1)^{\langle z+h, y\rangle},
$$

which is $2^{k-1}$ for $z=h$ and 0 otherwise.

Finally the Fourier Transform of $x \rightarrow x_{i}$ is

$$
z \rightarrow \sum_{y \in \mathbb{Z}_{2}^{k-1}} y_{i}(-1)^{\langle z, y\rangle}
$$

If $z_{j} \neq 0$ with $j \neq i$ then the terms in the above expression for $y$ and $y+e_{j}$ cancel each other out, so the Fourier Transform is 0 outside of $\left\{0, e_{i}\right\}$. For $z=0$ or $e_{i}$, the Fourier Transform is clearly $2^{k-2}$.

Combining these Fourier Transforms together gives the above form.

Then it follows that $f_{(a, b),\left(a_{1}, \ldots, a_{k-1}\right)}$ has a twin from which it has high distinguishing number.

Corollary 11. For distinct non-negative integers $a, b$ and positive integers $a_{1}, \ldots, a_{k-1}$, the multisets $f_{(a, b),\left(a_{1}, \ldots, a_{k-1}\right)}$ and $f_{(b, a),\left(a_{1}, \ldots, a_{k-1}\right)}$ have distinguishing number $k$. 
Proof. By Theorem 10, the fourier transforms $g_{1}=\hat{f}_{(a, b),\left(a_{1}, \ldots, a_{k-1}\right)}$ and $g_{2}=\hat{f}_{(b, a),\left(b_{1}, \ldots, b_{k-1}\right)}$ have identical support $\left\{0, e_{1}, e_{2}, \ldots, e_{k-1}, h\right\}$ and are equal except at $x=h$ for which we have $g_{1}(-x)=g_{2}(-x)$.

By Theorem 8, the distinguishing number of the two multisets is equal to the length of the minimum 0 -sum sequence $x_{1}, \ldots, x_{t}$ for which

$$
g_{1}\left(x_{1}\right) \ldots g_{1}\left(x_{t}\right) \neq g_{2}\left(x_{1}\right) \ldots g_{2}\left(x_{t}\right) .
$$

Clearly any such sequence must contain $h$ an odd number of times, leaving a 1-coordinate in each location that must be made up by a copy of $e_{i}$. Thus the shortest such sequence has length $k$ and is $e_{1}+e_{2}+\ldots+e_{k-1}+h=0$.

We will show that up to translation and linear maps on $\mathbb{Z}_{2}^{k-1}$, these are the only pairs of multisets of distinguishing number $k$. Say that two multisets $f_{1}$ and $f_{2}$ from $\mathbb{Z}_{2}^{k-1}$ of distinguishing number $k$ are in standard position if

$$
\hat{f}_{1}\left(e_{1}\right) \ldots \hat{f}_{1}\left(e_{k-1}\right) \hat{f}_{1}(h) \neq \hat{f}_{2}\left(e_{1}\right) \ldots \hat{f}_{2}\left(e_{k-1}\right) \hat{f}_{2}(h) .
$$

Theorem 12. For $k \geq 3$, if $f_{1}$ and $f_{2}$ are two multisets on $\mathbb{Z}_{2}^{k-1}$ of distinguishing number $k$, then there exists a bijective group homomorphism $\theta: \mathbb{Z}_{2}^{k-1} \rightarrow \mathbb{Z}_{2}^{k-1}$ such that $\theta f_{1}$ and $\theta f_{2}$ are in standard position.

Proof. By Theorem 8, there is a 0 -sum sequence of length $k, x_{1}, \ldots, x_{k}$ for which

$$
\hat{f}_{1}\left(x_{1}\right) \ldots \hat{f}_{1}\left(x_{k}\right) \neq \hat{f}_{2}\left(x_{1}\right) \ldots \hat{f}_{2}\left(x_{k}\right),
$$

and there is no shorter sequence.

In particular this means that $x_{1}, \ldots, x_{k}$ cannot be split into two shorter 0 -sum sequences (for they would both have equal sums), so $x_{1}, \ldots, x_{k-1}$ must be linearly independent and therefore must span $\mathbb{Z}_{2}^{k-1}$. As such there is a bijective group homomorphism $\theta: \mathbb{Z}_{2}^{k-1} \rightarrow \mathbb{Z}_{2}^{k-1}$ for which $\theta^{*} e_{i}=x_{i}$ for all $1 \leq i \leq k-1$.

Then by Lemma 6, we have

$$
\widehat{\theta f_{1}}\left(e_{1}\right) \ldots \widehat{\theta f_{1}}\left(e_{k-1}\right) \widehat{\theta f_{1}}(h) \neq \widehat{\theta g_{1}}\left(e_{1}\right) \ldots \widehat{\theta g_{1}}\left(e_{k-1}\right) \widehat{\theta g_{1}}(h) .
$$

Theorem 13. For $k \geq 3$, if $f_{1}$ and $f_{2}$ are two multisets on $\mathbb{Z}_{2}^{k-1}$ of distinguishing number $k$ in standard position, then there exist distinct non-negative integers $a, b$, positive integers $a_{1}, \ldots, a_{k-1}$ and elements $x_{1}, x_{2} \in \mathbb{Z}_{2}^{k-1}$ such that

$$
\begin{aligned}
& x_{1}+f_{1}=f_{(a, b),\left(a_{1}, \ldots, a_{k-1}\right)} \\
& x_{2}+f_{2}=f_{(b, a),\left(a_{1}, \ldots, b_{k-1}\right)}
\end{aligned}
$$

Proof. We know that

$$
\hat{f}_{1}\left(e_{1}\right) \ldots \hat{f}_{1}\left(e_{k-1}\right) \hat{f}_{1}(h) \neq \hat{f}_{2}\left(e_{1}\right) \ldots \hat{f}_{2}\left(e_{k-1}\right) \hat{f}_{2}(h),
$$

and there is no shorter such sequence. In particular, note that for all $x \in \mathbb{Z}_{2}^{k-1}, x+x=0$ is a shorter sequence, so $\hat{f}_{1}(x)^{2}=\hat{f}_{2}(x)^{2}$. 


\section{SET ReCOnstruction On THE Hypercube}

Now let $x$ be any element of $\mathbb{Z}_{2}^{k-1}$ other than $\left\{0, e_{1}, e_{2}, \ldots, e_{k-1}, h\right\}$, and let $I=\left\{i: 1 \leq i \leq k-1, x_{i}=\right.$ $1\}$ be the set of 1 coordinates of $x$. Note that by the choice of $x, 2 \leq p \leq k-2$. As such, it follows that $\left\{e_{i}: i \in I\right\} \cup\{x\}$ and $\left\{e_{i}: i \notin I\right\} \cup\{h, x\}$ are both 0-sum subsets of length at most $k-1$, and so it follows that

$$
\begin{aligned}
\prod_{i \in I} \hat{f}_{1}\left(e_{i}\right) \hat{f}_{1}(x) & =\prod_{i \in I} \hat{f}_{2}\left(e_{i}\right) \hat{f}_{2}(x) \text { and } \\
\prod_{i \notin I} \hat{f}_{1}\left(e_{i}\right) \hat{f}_{1}(h) \hat{f}_{1}(x) & =\prod_{i \notin I} \hat{f}_{2}\left(e_{i}\right) \hat{f}_{2}(h) \hat{f}_{2}(x) .
\end{aligned}
$$

Multiplying these together we get

$$
\prod_{i} \hat{f}_{1}\left(e_{i}\right) \hat{f}_{1}(h) \hat{f}_{1}(x)^{2}=\prod_{i} \hat{f}_{1}\left(e_{i}\right) \hat{f}_{2}(h) \hat{f}_{2}(x)^{2} .
$$

Since we know that $\hat{f}_{1}(x)^{2}=\hat{f}_{2}(x)^{2}$ and

$$
\hat{f}_{1}\left(e_{1}\right) \ldots \hat{f}_{1}\left(e_{k-1}\right) \hat{f}_{1}(h) \neq \hat{f}_{2}\left(e_{1}\right) \ldots \hat{f}_{2}\left(e_{k-1}\right) \hat{f}_{2}(h),
$$

it follows that $\hat{f}_{1}(x)=\hat{f}_{2}(x)=0$.

To summarize, $\hat{f}_{1}$ and $\hat{f}_{2}$ have no support outside of $\left\{0, e_{1}, \ldots, e_{k-1}, h\right\}$, we have $\hat{f}_{1}(x)^{2}=\hat{f}_{2}(x)^{2}$ for all $x$ and

$$
\hat{f}_{1}\left(e_{1}\right) \ldots \hat{f}_{1}\left(e_{k-1}\right) \hat{f}_{1}(h) \neq \hat{f}_{2}\left(e_{1}\right) \ldots \hat{f}_{2}\left(e_{k-1}\right) \hat{f}_{2}(h) .
$$

Now let $x_{1}=\sum_{\hat{f}_{1}\left(e_{i}\right)<0} e_{i}$ and $x_{2}=\sum_{\hat{f}_{2}\left(e_{i}\right)<0}$. Then for all $i, \widehat{f_{1}+x_{1}}\left(e_{i}\right)$ and $\widehat{f_{2}+x_{2}}\left(e_{i}\right)$ are positive numbers with the same square, and so are equal.

Thus $\widehat{f_{1}+x_{1}}$ and $\widehat{f_{2}+x_{2}}$ are of the form given in Theorem 10 except that we have not shown that $a, b$ are necessarily non-negative, or that any of $a, b, a_{1}, \ldots, a_{k-1}$ are necessarily integers.

To that end, note that $a=f_{1}+x_{1}(0), b=f_{2}+x_{2}(0)$ (and so they are indeed non-negative integers), and $a_{i}=f_{1}+x_{1}\left(e_{i}\right)-f_{2}+x_{2}(0)$, so is integral.

It follows that if we have $k$-distinguishable multisets, some element must appear $k$ times.

Theorem 14. For integers $k \geq 2$, if multisets $f$ and $g$ on $\mathbb{Z}_{2}^{k-1}$ have distinguishing number $k$, some element appears with multiplicity at least $k$ in $f$ or $g$.

Proof. By Theorem 13, $f$ and $g$ are translates of a linear image of some pair $f_{(a, b),\left(a_{1}, \ldots, a_{k-1}\right)}$ and $f_{(b, a),\left(a_{1}, \ldots, a_{k-1}\right)}$. The multiplicity of $h$ in these two multisets is $a+a_{1}+\ldots+a_{k-1}$ and $b+a_{1}+\ldots+a_{k-1}$. Since each $a_{i}$ is a positive integer, and $a$ and $b$ are distinct non-negative integers, one of these numbers is at least $k$.

This now allows us to prove our upper bound.

Corollary 15. For positive integers $n$ and $k$, if the reconstruction number of $\mathbb{Z}_{2}^{n}$ is at least $k$, then $2^{n+1-k} \geq k$ 
Proof. Suppose that the reconstruction number of $\mathbb{Z}_{2}^{n}$ is $t \geq k$. Then there exists two sets $S_{1}, S_{2} \subseteq \mathbb{Z}_{2}^{n}$ that are $t$-distinguishable, but not $t$-1-distinguishable.

Then by Theorem 9 , there exists a map $\theta: \mathbb{Z}_{2}^{n} \rightarrow \mathbb{Z}_{2}^{t-1}$ such that the multisets $\theta S_{1}, \theta S_{2}$ from $\mathbb{Z}_{2}^{t-1}$ are $t$-distinguishable, but not $t$-1-distinguishable.

Finally, by Theorem 14, there must be some element of $\mathbb{Z}_{2}^{t-1}$ that appears in $\theta S_{1}$ or $\theta S_{2}$ at least $t$ times. Since each element of $\mathbb{Z}_{2}^{t-1}$ only has $2^{n+1-t}$ inverse images, it follows that $2^{n+1-t} \geq t$.

Since $t \geq k$, we must have $2^{n+1-k} \geq 2^{n+1-t} \geq t \geq k$.

Tying everything together, we now have proved the precise value of the reconstruction number of the hypercube.

Corollary 16. The reconstruction number of $\mathbb{Z}_{2}^{n}$ is

$$
\left\lfloor n+1-\log _{2}\left(n+1-\log _{2}(n)\right)\right\rfloor .
$$

Proof. By Corollaries 5 and 15, the reconstruction number of $\mathbb{Z}_{2}^{n}$ is the maximum value of $k$ for which if $2^{n+1-k} \geq k$. By Theorem 1, this is $\left\lfloor n+1-\log _{2}\left(n+1-\log _{2}(n)\right)\right\rfloor$.

\section{Acknowledgments}

The author is grateful to the main anonymous reviewer for many helpful observations and tips.

\section{References}

[1] Noga Alon, Yair Caro, Ilia Krasikov and Yehuda Roditty Combinatorial Reconstruction Problems Journal of Combinatorial Theory, Series B, 47(2):153-161, 1989. 2

[2] Luke Pebody The Reconstructibility of Finite Abelian Groups. Combinatorics, Probability and Computing, 13(6):867-892, 2004. 1, 2, 6

[3] Jamie Radcliffe and Alex Scott Reconstructing subsets of $\mathbb{Z}_{n}$ Journal of Combinatorial Theory, Series A, 83(2):169-187, 1998. 2

\section{AUTHOR}

Luke Pebody

Rokos Capital Management

23 Savile Row

London, United Kingdom

luke [at] pebody [dot] org 